\title{
Reinforcing 3D Print Methacrylate Resin/Cellulose Nanocrystal Composites: Effect of Cellulose Nanocrystal Modification
}

\author{
Xinhao Feng, ${ }^{\mathrm{a}, \mathrm{b}}$ Zhihui Wu, ${ }^{\mathrm{a}, *}$ Yanjun Xie, ${ }^{\mathrm{c}}$ and Siqun Wang ${ }^{\mathrm{b}, *}$ \\ Cellulose nanocrystals (CNCs) were modified with methyl methacrylate \\ (MMA) to improve the properties of the resulting three-dimensional (3D) \\ stereolithography printed $\mathrm{CNC} /$ methacrylate $(\mathrm{MA})$ resin composites. The \\ dispersibility of the MMA-modified CNCs (MMA-CNCs) was substantially \\ improved, as evidenced by the limited precipitation in the MA solution. \\ Thermal gravimetry and differential scanning calorimetry measurements \\ showed that the pyrolytic temperature of the MMA-CNC was $110^{\circ} \mathrm{C}$ higher \\ than that of the CNCs; the pyrolytic temperature and glass transition \\ temperature of the resulting MMA-CNC/MA composites were higher than \\ those of the CNC/MA. The tensile strength and modulus of the MMA- \\ $\mathrm{CNC} / \mathrm{MA}$ composites were improved by up to $38.3 \mathrm{MPa}$ and $3.07 \mathrm{GPa}$, \\ respectively, compared to those of the CNC/MA composites. These results \\ demonstrated that the modification of CNC with MMA is a feasible \\ approach to substantially improve the mechanical properties and thermal \\ stability of the resulting MA-based composites.
}

Keywords: Methyl methacrylate; Cellulose nanocrystal; Grafting; Stereolithography; Thermal performances

Contact information: a: College of Furnishings and Industrial Design, Nanjing Forestry University, Nanjing, Jiangsu 210037, P. R. China; b: Center for Renewable Carbon, University of Tennessee, Knoxville, Tennessee 37996, USA; c: College of Material Science and Engineering, Northeast Forestry University, Harbin, Heilongjiang 150040, P. R. China;

*Corresponding authors:wzh550@sina.com; swang@utk.edu

\section{INTRODUCTION}

Three-dimensional (3D) stereolithography (3D-SL) printing is used to produce layer-laminated structures through curing liquid photopolymer resin under light irradiation (Becker and Gärtner 2008; Schaedler and Carter 2016). The 3D-SL printing process has shown higher resolution and accuracy for materials than the traditional processing techniques such as extrusion and compression molding (Cooke et al. 2003; Weng et al. 2016). Therefore, 3D-SL printing has been extensively applied in the areas of electronics (Zhang et al. 1999; Lee et al. 2008), tissue engineering (Melchels et al. 2010; Suntornnond et al. 2017), and polymer engineering (Nguyen et al. 2005; Mao et al. 2018). Because of the specific requirements, only limited types of resins, such as acrylate, epoxy, and polyurethane (Melchels et al. 2010), are commercially available for the 3D-SL processing. Materials containing these resins as a base often exhibit undesirable embrittlement caused by the formation of a highly crosslinked network (Lin et al. 2015), and the preferable properties are time dependent in the epoxy- and acrylate-resin matrix after printing (Crivello and Reichmanis 2014; Yang et al. 2018). In addition, polymerization of the resin is uncontrollable and incomplete during the printing and post-curing (at low temperature) processes (Lin et al. 2007; Melchels et al. 2010; Qin et al. 2010; Yang et al. 2018), which 
results in insufficient mechanical strength (Moon et al. 2014; Lin et al. 2015), dimensional instability (Esposito Corcione et al. 2004; Xu and Chen 2015), and low flexibility (Vatani et al. 2015).

Reinforcements, such as cellulose nanocrystal (CNC), have been used to overcome the problems of 3D-SL-printed materials because of its high specific strength, large aspect ratio, and renewability (Kedzior et al. 2016; Yang et al. 2018). Various types of cellulose, such as fibers, CNC, and bacterial nanocellulose, have exhibited potential to transfer stresses between deformed matrices and improve the properties of 3D-printed composites (Kramer et al. 2006; Farahani et al. 2012; Chiappone et al. 2014; Kim et al. 2014). They have been applied to improve the mechanical properties of methacrylate-based photoreactive resins (Shanmuganathan et al. 2009; Singha and Thakur 2009; Feng et al. 2017). However, the mixture of CNC and resin must be continuously stirred before sample fabrication due to the lack of adequate compatibility of hydrophilic cellulose in the hydrophobic resin matrix. As a result, aggregation of cellulose in the matrix and phase separation were found both in the mixture of $\mathrm{CNC}$ and resin before printing and in the printed nanocomposites, resulting in insufficient interfacial interaction and weak reinforcement efficacy of CNC (Singha and Thakur 2009; Feng et al. 2017; Feng et al. 2018; Yang et al. 2018).

To solve this issue, a feasible strategy is to obtain a hydrophobic characteristic of CNC through surface modification. The best modifying agent should be both reactive with $\mathrm{CNC}$ and compatible to the methacrylate (MA) resin matrix. The modification of cellulose with hydrophobic monomers, such as methyl methacrylate (MMA), has been shown to improve the compatibility between cellulose and the photopolymer resin (Kedzior et al. 2016). Cotton cellulose, bamboo cellulose, and nanofibrillated cellulose (NFC) were modified by the acrylic monomers in an aqueous solution via free radical reaction, and the resulting cellulosic materials were found to be more hydrophobic and thermally stable compared to the unmodified ones, and grafting with acrylic monomers resulted in no changes to the microstructure of cellulose materials (Littunen et al. 2011; Wan et al. 2011; Routray and Tosh 2012). The modified NFC and CNC were used as a reinforcement particles within a poly(methyl methacrylate) (PMMA) matrix to fabricate nanocomposites, and the interfacial adhesion between NFC/CNC and PMMA was improved (Littunen et al. 2013; Kedzior et al. 2016). However, the mechanical properties and thermal stability of the resulting composites decreased due to the aggregation of the MMA grafted NFC/CNC in the PMMA matrix (Littunen et al. 2013).

The authors have previously used CNCs to reinforce 3D-printed methacrylate composites and found that the addition of $\mathrm{CNCs}$ below $0.5 \%$ did not influence the tensile properties; incorporating higher $\mathrm{CNC}$ amounts resulted in a decrease in the tensile strength (8\%) and elongation (55\%) (Feng et al. 2017). The decrease is mainly attributable to the uneven dispersion of CNCs in the MA matrix and the failure of interfacial adhesion between the CNC and MA matrix due to the different polarity of the CNC and MA molecule. Therefore, in this study, the chemical modification of CNC via MMA grafting was performed to improve its compatibility with the MA molecule. The dispersibility of $\mathrm{CNC}$ both before and after modification was investigated by dispersing CNCs in the MA matrix, and the thermal stability of $\mathrm{CNC}$ was analyzed by the thermal analyzer. The reinforcement effect of MMA-modified CNC (MMA-CNC) in the printed composite was substantially exploited, as evidenced by its mechanical and thermal properties, compared with those of CNC/MA composites. 


\section{EXPERIMENTAL}

\section{Materials}

Aqueous CNC suspensions with a dry CNC content of $6.3 \mathrm{wt} \%$ were obtained from the University of Maine (Orono, ME, USA). They were mainly produced by using sulfuric acid to hydrolyze the amorphous regions of the cellulose polymer, leaving the acid-resistant crystals as a product. Subsequently the crystals were purified by diluting and neutralizing the acid and then separating the soluble chemicals from the insoluble CNC. The diameter and length of the CNC particles were from $20 \mathrm{~nm}$ to $40 \mathrm{~nm}$ and from $150 \mathrm{~nm}$ to $250 \mathrm{~nm}$, respectively.

The MA, a commercial product, was purchased from a 3D printing manufacturer (Formlabs Inc., Somerville, MA, USA). It mainly consisted of an oligomer and photoinitiator. Acetone, MMA, cerium (IV) ammonium nitrate (CAN), and nitric acid were supplied by Fisher Scientific Company LLC (Pittsburgh, PA, USA) and used as received. All solvents were analytical reagent grade.

\section{Graft copolymerization}

The aqueous $\mathrm{CNC}$ suspension was adjusted to a $\mathrm{pH}$ of 1 with diluted nitric acid. The CAN was added as an initiator, and then the obtained mixture was magnetically stirred for $30 \mathrm{~min}$ at $45{ }^{\circ} \mathrm{C}$. The MMA was gradually added over $30 \mathrm{~min}$ and then stirred for 60 min to allow a complete grafting reaction between CNC and MMA (Fig. 1). The initiator concentration was $0.004 \mathrm{mmol} / \mathrm{cm}^{3}$, and the amount of MMA was $40 \mathrm{mmol}$ per $\mathrm{g}$ of dry CNC.

The reaction mixture was stirred and then centrifuged (4000 rpm, $20 \mathrm{~min}$ ) with acetone three times to remove the homopolymer, initiator, and nitric acid (Littunen et al. 2011). The MMA-modified CNC (abbreviated as MMA-CNC in this context) was dispersed in acetone solution and then sealed for further experiments.

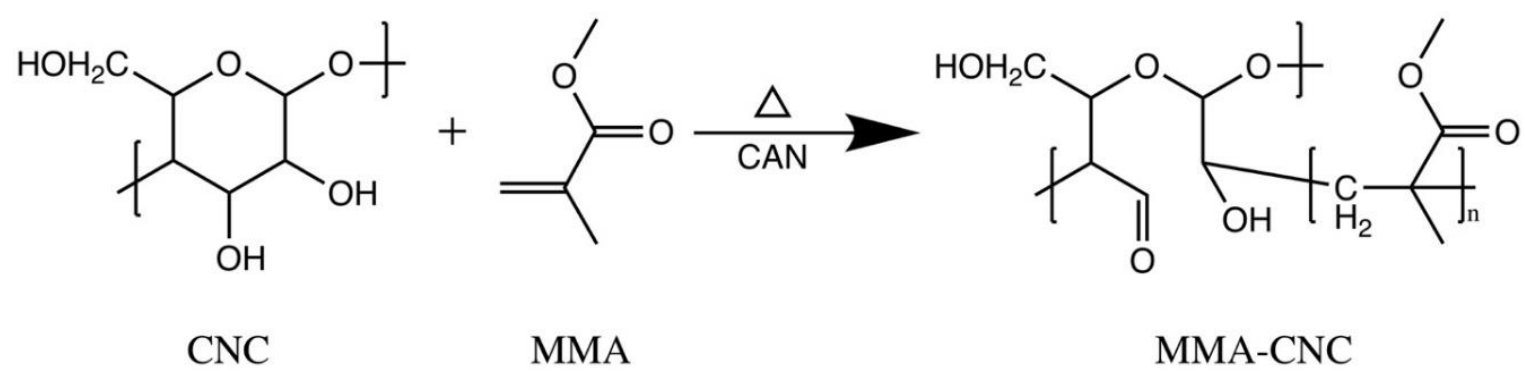

Fig. 1. Schematic of MMA grafting onto a CNC and polymerization

Preparation of MA-based composites by $3 D$ printing

The MMA-CNCs or CNCs were directly mixed with MA at a content of $0 \mathrm{wt} \%, 0.5$ $\mathrm{wt} \%, 1 \mathrm{wt} \%, 2 \mathrm{wt} \%$, and $4 \mathrm{wt} \%$. Subsequently, the mixture was magnetically stirred for $10 \mathrm{~min}$ and then ultrasonically treated at $300 \mathrm{~W}$ for $6 \mathrm{~min}$. The resulting mixture was 3D printed to prepare the samples through the process previously described (Feng et al. 2017). A total of $50 \%$ of the printed samples were treated using a post-curing process in an oven at $130{ }^{\circ} \mathrm{C}$ for $40 \mathrm{~min}$. 


\section{Methods}

Fourier transform infrared (FTIR) analysis

The FTIR spectra of unmodified and modified CNCs were analyzed using a FTIRattenuated total reflectance (ATR) spectrometer (Spectrum One; PerkinElmer Corporation, Waltham, MA, USA) at $25{ }^{\circ} \mathrm{C}$. The samples were placed onto the diamond crystal of an ATR accessory. Data were collected over the spectral range of $400 \mathrm{~cm}^{-1}$ to $4000 \mathrm{~cm}^{-1}$ with a resolution of $4 \mathrm{~cm}^{-1}$. A total of 20 scans were applied for each sample.

\section{Scanning transmission electron microscopy (STEM) analysis}

The images of the MMA-CNCs and CNCs were analyzed by STEM, using a Zeiss Auriga dual beam SEM/Focus ion beam (FIB) (crossbeam 550; Carl Zeiss AG, Jena, Germany) with an accelerating voltage of $30 \mathrm{kV}$. Each sample for the STEM analysis was prepared by depositing one drop of MMA-CNC or CNC sample $(0.05 \mathrm{wt} \%)$ onto a standard holey-carbon-film-covered copper grid (CF200-CU; Electron Microscopy Sciences, Hatfield, PA, USA), followed by the removal of excess liquid from the bottom of the grid and drying at ambient temperature before loading onto the microscope. The diameter and length of at least 100 particles were measured from five randomly selected STEM images. To investigate the dispersing durability of MMA-CNCs and CNCs in the MA matrix, the images of mixtures of MMA-CNC/MA and CNC/MA were taken at intervals of $0,5,10$, 15,20 , and 60 days.

\section{Tensile testing}

The tensile strength, tensile modulus, and elongation of the printed MMACNC/MA and CNC/MA composites were tested at a speed of $1 \mathrm{~mm} / \mathrm{min}$ according to ASTM D638 (2014) using a universal testing machine (Model 5567; Instron ${ }^{\circledR}$, Norwood, MA, USA). The dumbbell-shaped samples with dimensions of $63.5 \mathrm{~mm} \times 9.53 \mathrm{~mm} \times 3.2$ $\mathrm{mm}(\mathrm{L} \times \mathrm{W} \times \mathrm{T})$ were placed at $23 \pm 2{ }^{\circ} \mathrm{C}$ and $50 \pm 5 \%$ relative humidity for $24 \mathrm{~h}$. A total of five duplicates were tested for each sample.

\section{Thermal stability and performances}

The thermal stability of MMA-CNCs, CNCs, and 3D-printed MMA-CNC/MA and CNC/MA composites were determined using the Perkin-Elmer 7 series thermal analyzer (PerkinElmer, Inc., Shelton, CT, USA). Each sample (approximately $8 \mathrm{mg}$ ) was heated at a heating rate of $10{ }^{\circ} \mathrm{C} / \mathrm{min}$ from $25^{\circ} \mathrm{C}$ to $600{ }^{\circ} \mathrm{C}$ in a dry air atmosphere. Three duplicates were measured for each sample.

The thermal performances of the composites were measured using a differential scanning calorimeter (DSC; Diamond, PerkinElmer Corporation, Waltham, MA, USA). First, a sample of approximately $5 \mathrm{mg}$ was heated from $25^{\circ} \mathrm{C}$ to $210{ }^{\circ} \mathrm{C}\left(10^{\circ} \mathrm{C} / \mathrm{min}\right)$ to remove the residual moisture and erase any thermal history. The temperature was held at $210{ }^{\circ} \mathrm{C}$ for $5 \mathrm{~min}$ and then cooled to $25^{\circ} \mathrm{C}\left(10^{\circ} \mathrm{C} / \mathrm{min}\right)$. Next, the sample was reheated from $25^{\circ} \mathrm{C}$ to $210{ }^{\circ} \mathrm{C}\left(10{ }^{\circ} \mathrm{C} / \mathrm{min}\right)$. A nitrogen flow of $20 \mathrm{~mL} / \mathrm{min}$ was applied throughout the DSC process to prevent oxidation. The glass transition temperature $\left(T_{\mathrm{g}}\right)$ was determined from the endothermic transition in the second scan, and the thermal polymerization temperature $\left(T_{\mathrm{p}}\right)$ and enthalpy $(\Delta H)$ were determined by the corresponding exothermic transition and peak area in the first scan, respectively. 


\section{RESULTS AND DISCUSSION}

\section{Characteristics of the MMA-modified CNC}

The CNCs displayed typical absorption bands such as $\mathrm{OH}$ stretching at $3336 \mathrm{~cm}^{-1}$, $\mathrm{CH}$ stretching at $2897 \mathrm{~cm}^{-1}$, and bound water at $1646 \mathrm{~cm}^{-1}$ (Fig. 2) (Routray and Tosh 2012; Tran et al. 2016). After modification with MMA, the MMA-CNCs exhibited new bands, such as asymmetrical $\mathrm{CH}_{3}$ stretching at $2951 \mathrm{~cm}^{-1}, \mathrm{C}=\mathrm{O}$ stretching at $1704 \mathrm{~cm}^{-1}$, asymmetric COO- stretching at $1538 \mathrm{~cm}^{-1}$, and $\mathrm{CH}_{2}$ rocking at $774 \mathrm{~cm}^{-1}$, which were assigned to the grafting of MMA onto CNCs and the polymerized MMA (Dai Prè et al. 2013; Anžlovar et al. 2016; Tran et al. 2016). This result confirmed that MMA was successfully grafted onto the CNC surface. The band intensity of MMA-CNCs located at $3336 \mathrm{~cm}^{-1}$ was considerably lower than that of the CNCs, and the band at $1646 \mathrm{~cm}^{-1}$ disappeared after modification. These variations were generally related to the modification of the $\mathrm{H}$-bonding between the $\mathrm{C}=\mathrm{O}$ group and the free $-\mathrm{OH}$ group in the MMA-CNC network, resulting in the hydrophobic characteristics of the MMA-CNCs (Sain et al. 2015; Tran et al. 2016).

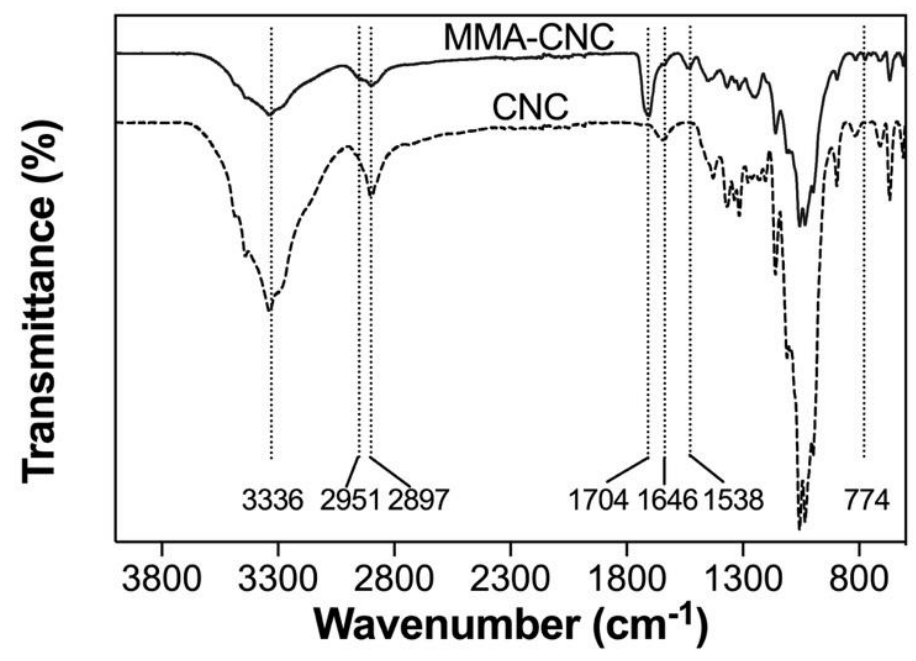

Fig. 2. FTIR spectrums of CNC unmodified and modified with MMA

After MMA modification, the dimensions of MMA-CNC were smaller than that of $\mathrm{CNC}$; the length and diameter was $155.2 \mathrm{~nm} \pm 24.3 \mathrm{~nm}$ and $27.7 \mathrm{~nm} \pm 4.4 \mathrm{~nm}$, respectively, for MMA-CNC, and $222.7 \mathrm{~nm} \pm 46.4 \mathrm{~nm}$ and $29.9 \mathrm{~nm} \pm 8.5 \mathrm{~nm}$, respectively, for CNC (Fig. 3). The CNC aggregation was clearly observed from the longitudinal direction (Figs. $3 \mathrm{a}$ and $3 \mathrm{~b}$ ), and the diameter of the CNCs was narrowly dispersed in the range of $20 \mathrm{~nm}$ to $35 \mathrm{~nm}$. When MMA was grafted onto the CNCs, the length of MMA-CNCs were shorter than $\mathrm{CNCs}$, and the diameter also decreased (Figs. 3c and 3d), indicating that the hydrogen bonds and van der Waals forces between the CNCs was substituted by chemical bonds formed between the CNCs and MMA. Moreover, the rupture of CNC molecular chains occurred through incorporation of the initiator and acid medium during grafting copolymerization (Canche-Escamilla et al. 2002). More importantly, the MMA-CNC was evenly dispersed in the solution without obvious aggregation, resulting in the homogenous distribution of MMA-CNC in the MA resin, as was previously discussed. 

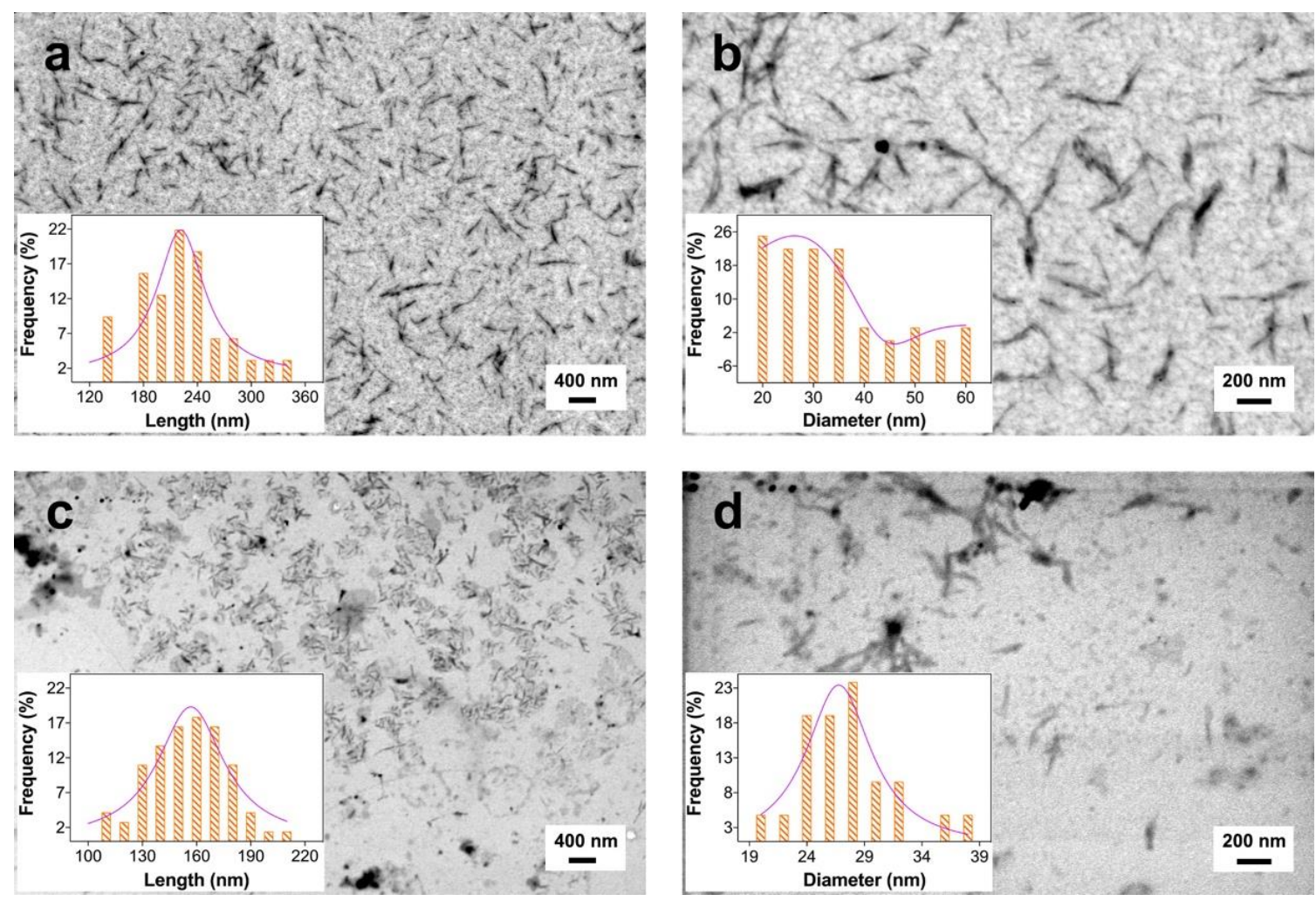

Fig. 3. STEM morphology of CNCs (a, b) and MMA-CNCs (c, d); inset graphs are the length $(a, c)$ and diameter $(b, d)$ of CNCs and MMA-CNCs

The CNCs exhibited a minor weight loss at approximately $80{ }^{\circ} \mathrm{C}$ caused by the evaporation of moisture (Fig. 4) (Sain et al. 2015). The CNCs displayed a one-step degradation that started at $225{ }^{\circ} \mathrm{C}\left(T_{5} \%\right.$, temperature at $5 \%$ weight loss $)$ and obtained peak degradation at $315{ }^{\circ} \mathrm{C}$ ( $T_{\max 1}$, peak degradation temperature). However, MMA-CNCs showed a two-step degradation, with an initial degradation at $189{ }^{\circ} \mathrm{C}$ and two degradation peaks at $203{ }^{\circ} \mathrm{C}\left(T_{\max 1}\right)$ and $420{ }^{\circ} \mathrm{C}\left(T_{\max 2}\right)$.

The initial temperature of MMA-CNC, which was attributed to the elimination of coordinated water molecules (possibly through hydrogen bonds) and the decomposition of weak carbon-carbon bonds between units linked head-to-head (Sain et al. 2012; Tran et al. 2016), was lower than that of $\mathrm{CNC}$, and the $T_{\max 1}$ shifted to a lower temperature in the MMA-CNC relative to that observed for the default CNC. This result was explained by the relatively low thermal stability of MMA compared to unmodified CNC and the degradation of $\mathrm{CNC}$ caused by both the oxidation of $\mathrm{CNC}$ and the rupture of $\mathrm{CNC}$ molecular chains during grafting in the initiator and acid medium (Canche-Escamilla et al. 2002; Tran et al. 2016).

However, a new degradation peak $\left(T_{\max 2}\right)$ was observed in the MMA-CNCs that was higher than the $T_{\max 1}$ of CNCs, and the weight of MMA-CNCs $(60 \%)$ did not change from $250{ }^{\circ} \mathrm{C}$ to $400{ }^{\circ} \mathrm{C}$. The $\mathrm{CNCs}$ exhibited peak degradation and maximum weight loss (down to 20\%), which indicated that the thermal stability of the CNCs were noticeably improved by graft copolymerization with MMA. 


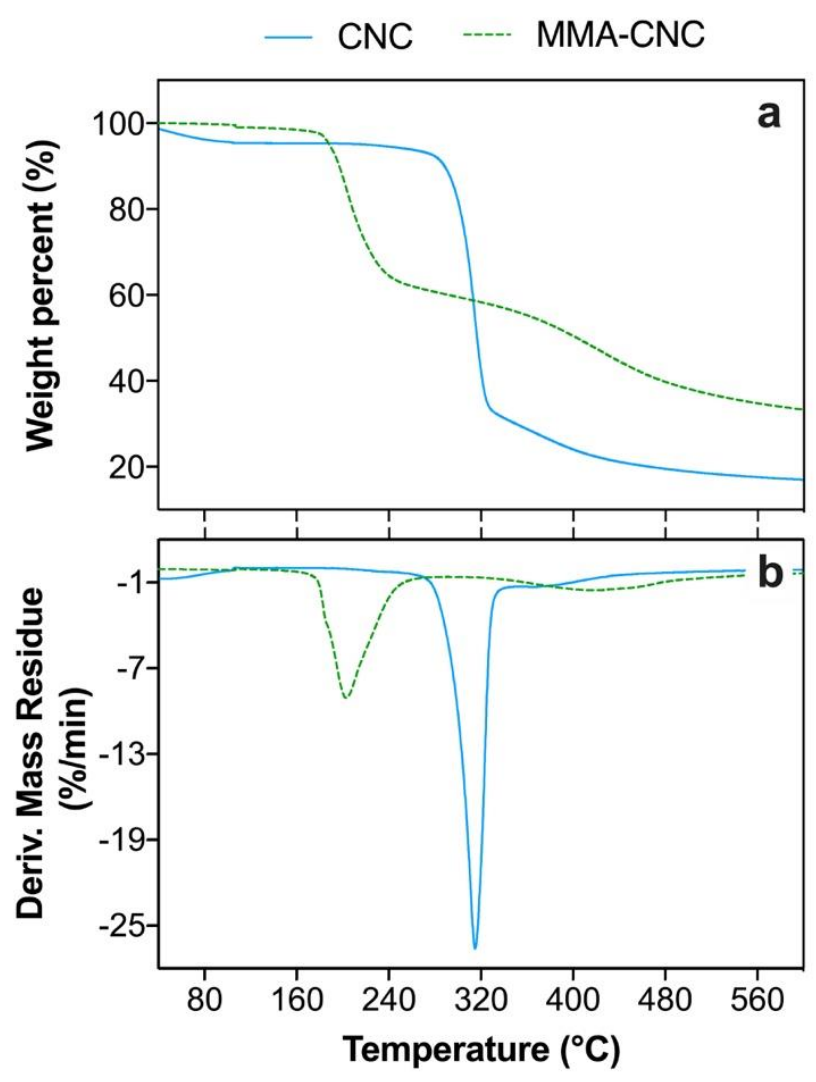

Fig. 4. TG (a) and DTG (b) of CNCs and MMA-CNCs

\section{Phase Separation of CNC in MA Resin}

The phase states of the MMA-CNC/MA and CNC/MA mixtures after different days storing are shown in Fig. 5. Both the MMA-CNC/MA and CNC/MA mixtures showed a homogenous and milky solution after ultrasonication (Fig. 5a). However, the CNC began to precipitate from the mixture after two days storing (not shown), and a clear MA resin was observed on the top of the CNC/MA mixture after 5 days storing (Fig. 5b, left). The MMA-CNC was not precipitated from the mixture until 10 days storing (Fig. 5c, right), at which point a pale-white MA resin containing few MMA-CNCs was observed, and the clear and transparent MA resin was separated from the mixture via the precipitation of CNCs (Fig. 5c, left). From 15 days to 60 days storing, the height of the separated MA resin linearly increased in the CNC/MA mixture (Fig. $5 \mathrm{~d}$ to $5 \mathrm{f}$, left), and at 60 days the $\mathrm{CNCs}$ were totally precipitated from half of the MA resin. As a result, more than half of the clear and transparent MA resin appeared above the rest of the mixture. However, the separation of MMA-CNC from the mixture was slow and incomplete with increasing storing time (Fig. 5d to 5f, right) because the height of the separated MA resin slowly increased and leveled off from 20 days to 60 days, and the color of the separated MA resin was still palewhite, indicating that a low amount of MMA-CNCs were still included in the separated MA resin because of the good dispersibility of MMA-CNCs in the MA resin (Littunen $e t$ al. 2011). Therefore, the CNC obtained higher dispersibility in the mixture with MA resin through MMA grafting copolymerization compared with that of the unmodified CNCs. 

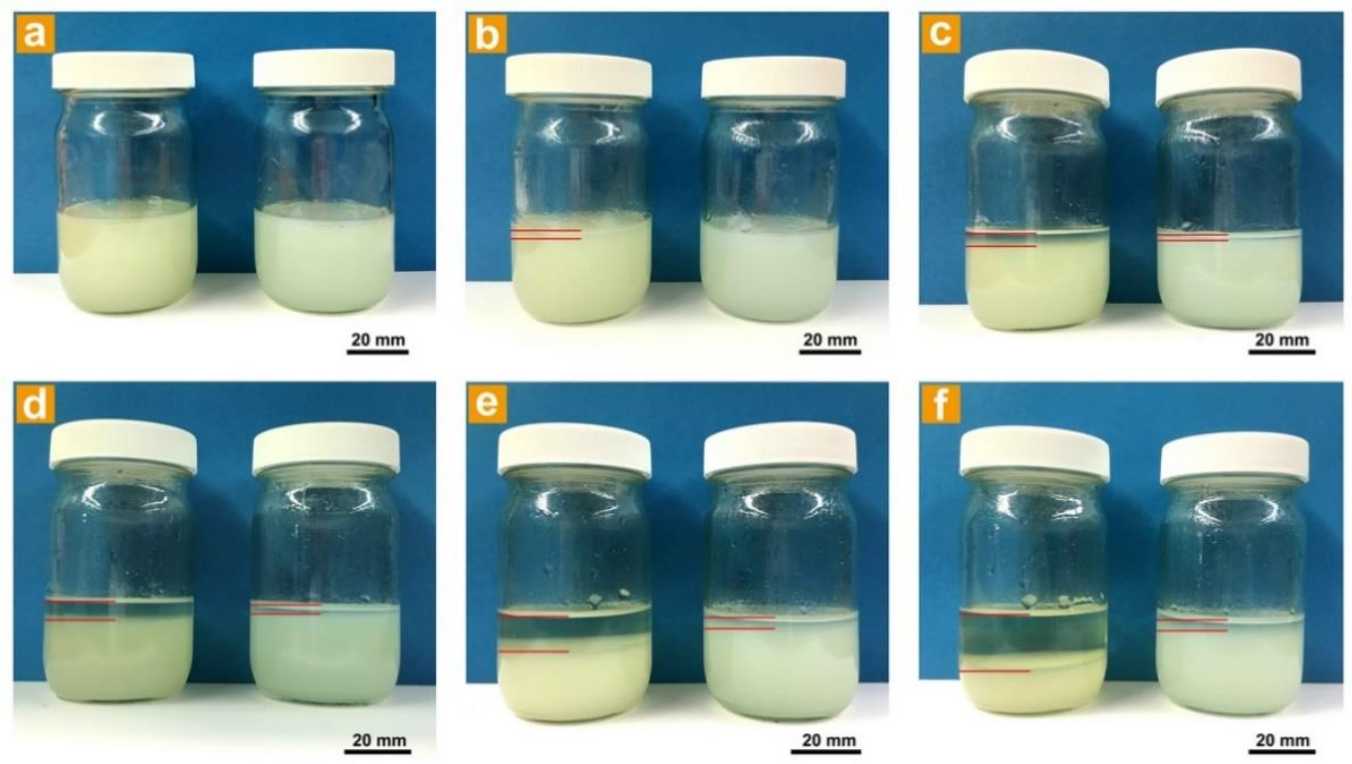

Fig. 5. Photographs of $1 \mathrm{wt} \% \mathrm{MMA}-\mathrm{CNC} / \mathrm{MA}$ (right) and $1 \mathrm{wt} \% \mathrm{CNC} / \mathrm{MA}$ (left) mixtures after 0 days (a), 5 days (b), 10 days (c), 15 days (d), 20 days (e), and 60 days (f)

\section{Effect of CNC Modification on the Mechanical Properties of the Composites}

Through grafting MMA onto the CNCs, the MMA-CNC/MA composites obtained a superior mechanical property and structure formability compared to those of the CNC/MA composites, both before and after post-cure (Fig. 6). With increased MMA-CNC content, the tensile strength increased at $0.5 \%$ and decreased from $1 \%$ to $4 \%$ for MMA$\mathrm{CNC} / \mathrm{MA}$ composites, indicating that an almost ideal dispersion of MMA-CNC in the MA matrix was obtained at $0.5 \%$ MMA-CNC, resulting in better interactions between the MMA-CNCs and the MA matrix through molecular entanglement and miscibility (CancheEscamilla et al. 2002). However, the tensile strength of CNC/MA composites decreased with increasing $\mathrm{CNC}$ content due to the agglomerates of $\mathrm{CNC}$ particles that served as weak points in the composites, resulting in poor compatibility and dispersibility of CNC in the MA matrix, which was observed in the mixture of CNC/MA (Fig. 5).

The tensile modulus increased both in the MMA-CNC/MA and CNC/MA composites because of the high elastic modulus of MMA-CNC and CNC (Tran et al. 2016). It leveled off with increasing MMA-CNC or CNC content from $0.5 \%$ to $4 \%$ for MMACNC/MA composites and from $0.5 \%$ to $2 \%$ for CNC/MA composites (Figs. 6c and 6d). A similar result in which a substantial increase in the Young's modulus was obtained at a low PMMA-NCC content $(0.005 \%$ and $0.01 \%)$ was also reported in a previous study (Anžlovar et al. 2016). However, for the same MMA-CNC or CNC content, the increment of tensile modulus for MMA-CNC/MA composites and CNC/MA composites after post-cure was more obvious than those before post-cure, and the MMA-CNC/MA composites obtained a higher increment than the $\mathrm{CNC} / \mathrm{MA}$ composites. This result was attributed to the further interaction of the MMA-CNCs or CNCs with the MA matrix that occurred during postcure (Feng et al. 2017). The MMA-CNCs were more efficient and reactive than the default CNCs according to the higher increment of MMA-CNC/MA composites (48\%) than the CNC/MA composites (12\%), which was confirmed by the polymerization temperature and enthalpy from the DSC measurement. 

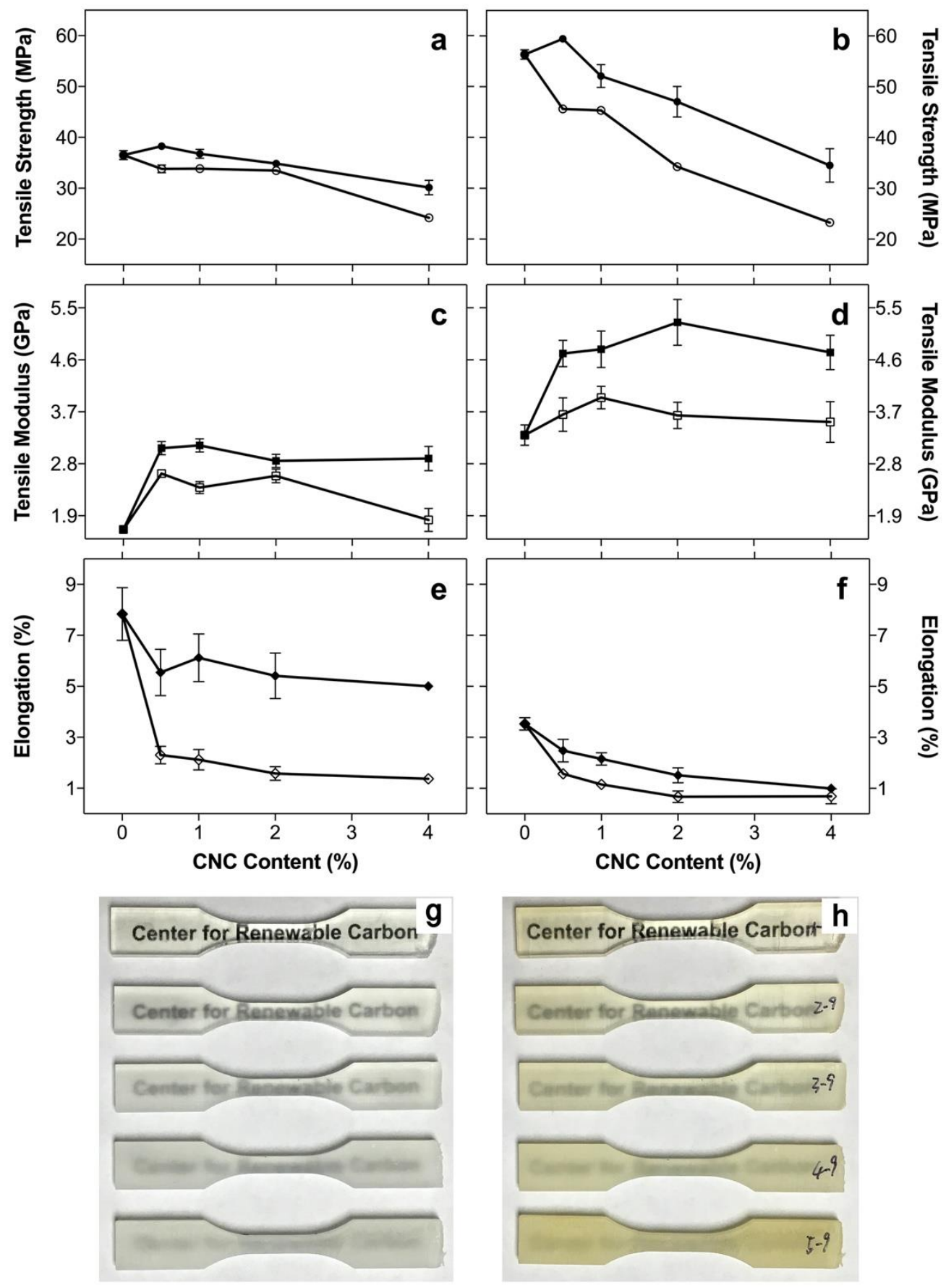

Fig. 6. Tensile strength $(a, b)$, tensile modulus $(c, d)$, elongation $(e, f)$, and photographs $(g, h)$ of 3D-printed MMA-CNC/MA (solid symbol, $g$ and $h$ ) and CNC/MA (hollow symbol) composites before $(a, c, e$, and $g)$ and after $(b, d, f$, and $h)$ post-curing process

The elongation of composites decreased with increased MMA-CNC or CNC content, both before and after post-cure; this was attributed to the uneven dispersion of MMA-CNCs, CNCs in the MA matrix, and the limited movement of MA molecular chains in the rigid MA matrix (Tsai et al. 2016; Feng et al. 2017). However, before post-cure, the decrease of elongation leveled off when the MMA-CNC or CNC content increased from 
$0.5 \%$ to $4 \%$ by considering the value deviations of elongation; there were $29 \%$ and $76 \%$ decreases for MMA-CNC/MA and CNC/MA composites, respectively, compared to that of the pure MA composite. Moreover, the decrease after post-cure was more profound in the CNC/MA composites than that of the MMA-CNC/MA composites. This implied that the MMA-CNC generated more plasticization in the composite than CNC, due to the higher dispersibility and compatibility of MMA-CNCs in the matrix than CNCs, which was caused by efficient interaction and entanglement of MMA-CNCs with the MA molecular chains (Canche-Escamilla et al. 2002).

From the photographs of a printed sample, both MMA-CNC/MA (Figs. 6g and 6h) and CNC/MA (not shown) composites formed dumbbell-shaped structures. With longer printing time, the CNCs aggregated into clusters of nanoparticles that hindered the polymerization of MA molecules, and precipitated in the MA matrix to show an uneven transparency in the sample. However, MMA-CNCs were evenly dispersed in the MA (Fig. 5 , right) and did not show any precipitation during printing. The printed MMA-CNC/MA composites both before and after post-cure also showed uniform transparency in the same sample, indicating well-dispersed MMA-CNC in the printed structure (Figs. 6g and 6h) and good printability of MMA-CNC within the MA resin.

\section{Effect of CNC Modification on the Thermal Stability of the Composites}

The TG of MMA-CNC/MA and CNC/MA composites exhibited two-step degradations, both before and after post-cure (Figs. 7 and 8). The first degradation ranged between $370{ }^{\circ} \mathrm{C}$ to $380{ }^{\circ} \mathrm{C}$ and corresponded to the decomposition of the relatively weak carbon-carbon bonds between MMA units linked head-to-head. The other degradation occurred between $460{ }^{\circ} \mathrm{C}$ to $470{ }^{\circ} \mathrm{C}$ and was attributed to the cleavage of terminal vinyl chain-end units; the most stable bonds between MMA units were linked head-to-tail (Sain et al. 2012; Tran et al. 2016). The temperature at $5 \%$ weight loss $\left(T_{5} \%\right)$ of both composites before post-cure increased between $0.5 \%$ and $2 \%$ MMA-CNC or CNC content and decreased above $4 \%$ compared to those of the pure MA composites (Figs. 7a and 7b). However, the $T_{5} \%$ of MMA-CNC/MA composites was higher than that of CNC/MA composites at the same CNC level because of the higher dispersibility and compatibility of MMA-CNCs in the MA matrix than CNCs, as discussed above. The temperature at $95 \%$ weight loss $\left(T_{95} \%\right)$ before post-cure exhibited different behavior from $T_{5} \%$, in which the $T_{95 \%}$ of MMA-CNC/MA composites increased over the entire range of MMA-CNC content. No difference was found in the CNC/MA composites. Two peak decomposition temperatures ( $T_{\max 1}$ and $T_{\max 2}$ ) showed similar performance as $T_{5} \%$ and $T_{95 \%}$ (Figs. $7 \mathrm{c}$ and $7 \mathrm{~d}$ ). The $T_{\max 1}$ of MMA-CNC/MA composites was higher than that of CNC/MA composites at $0.5 \%$ and $1 \%$ MMA-CNC content, and a more considerable increase in $T_{\max 2}$ was observed in the MMA-CNC/MA composites compared to that of the CNC/MA composites. This indicated the thermal stability of MA composites was improved by MMA-CNC via its good dispersibility and compatibility in the MA matrix. In contrast, the improvement was dominated by $T_{\max 2}$. As a result, a more obvious increment was found in $T_{\max 2}$ than in $T_{\max 1}$.

Compared to the thermal performances of MA composites before post-cure, those after post-cure exhibited completely different behaviors (Fig. 8). The $T_{5} \%, T_{95 \%}, T_{\max }$, and $T_{\max 2}$ of the MMA-CNC/MA composites increased from $0.5 \%$ to $2 \%$ MMA-CNC content compared to those of pure MA composites, whereas a decrease was found for the CNC/MA composites. 


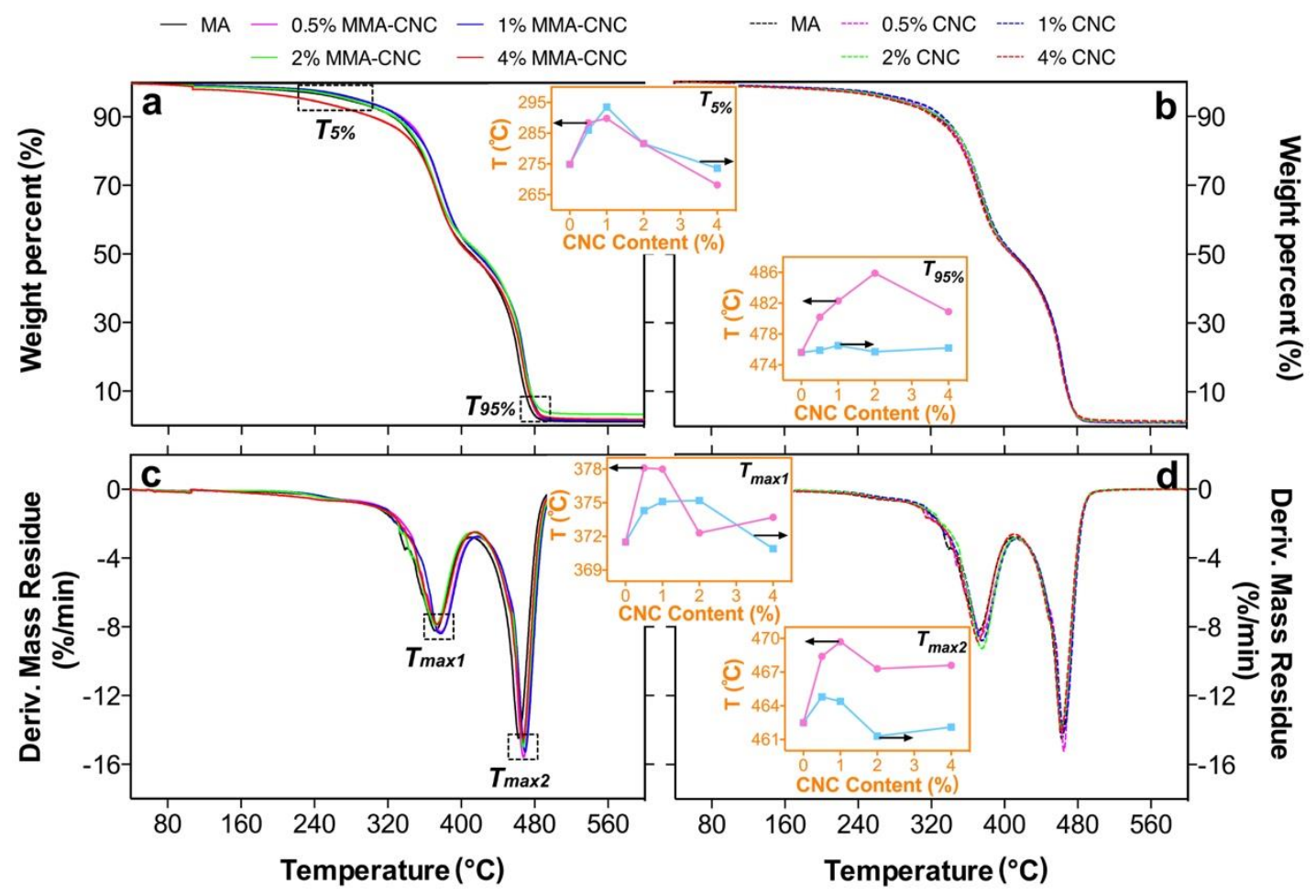

Fig. 7. TG (a, b) and DTG (c, d) of MMA-CNC/MA (a, c) and CNC/MA (b, d) composites before post-curing; inset images are the $T_{5 \%}, T_{95}, T_{\max 1}$, and $T_{\max 2}$ values corresponding to MMA-CNC or CNC content of the MMA-CNC/MA (round pink) and CNC/MA (blue square) composites

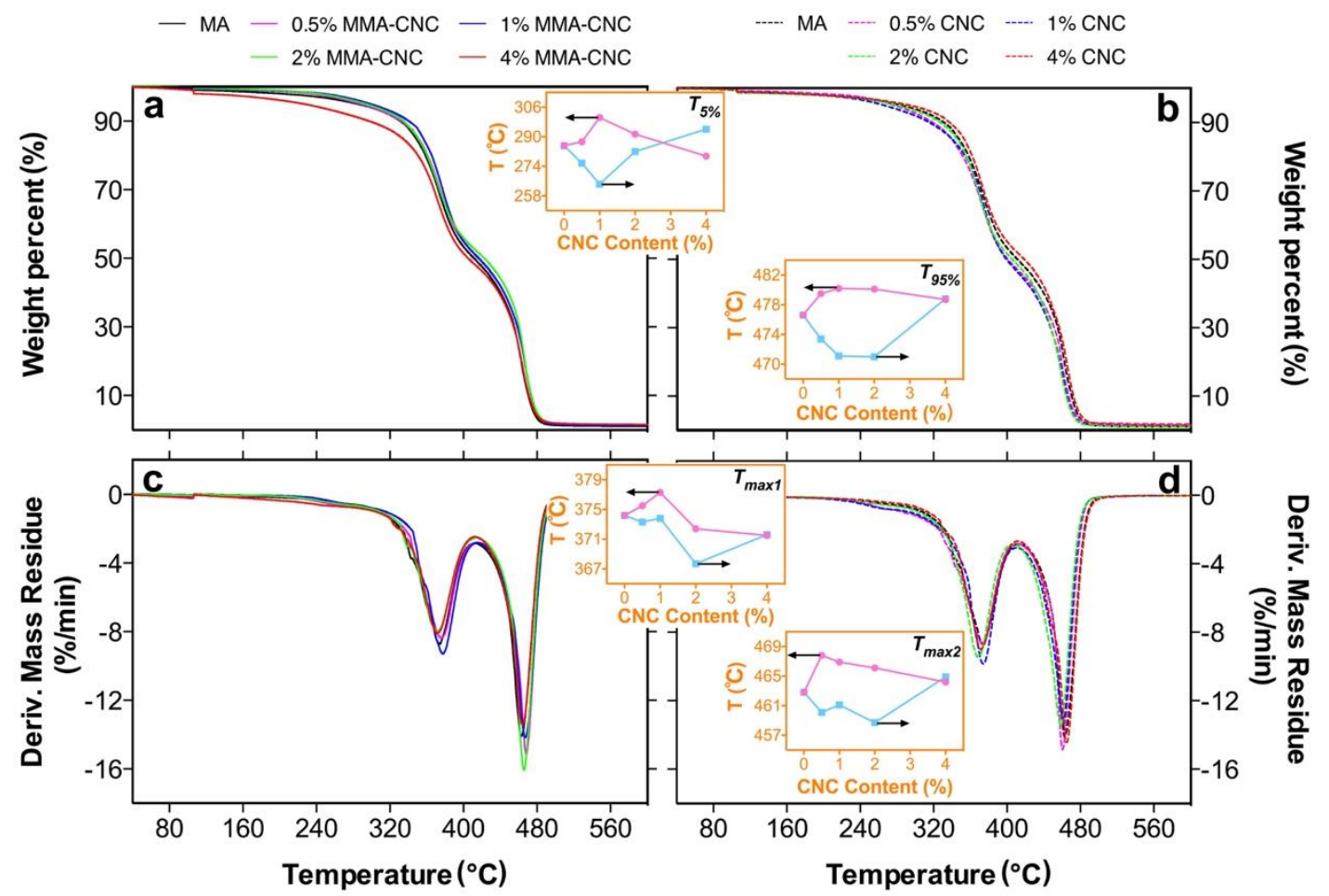

Fig. 8. TG (a, b) and DTG (c, d) of MMA-CNC/MA (a, c) and CNC/MA (b, d) composites after post-curing; inset images are $T_{5 \%}, T_{95}, T_{\max 1}$, and $T_{\max 2}$ values corresponding to the MMA-CNC or CNC contents of MMA-CNC/MA (round pink) and CNC/MA (blue square) composites 
This result demonstrated that the MMA-CNC/MA composites underwent thermal degradation at a higher temperature than the CNC/MA composites, which was attributed to more efficient hydrogen bonding interaction between the MMA-CNC hydroxyl groups and the carbonyl groups in the MA matrix. This facilitated the miscibility of the polymer blends and the high dispersibility of MMA-CNCs in the MA matrix that improved the entanglement between the MMA-CNCs and the MA molecular chains (Kuo 2008; Dong et al. 2012).

The glass transition temperature $\left(T_{\mathrm{g}}\right)$, thermal polymerization temperature $\left(T_{\mathrm{p}}\right)$, and enthalpy $(\Delta H)$ of both composites before and after post-cure are shown in Table 1 . The $T_{\mathrm{g}}$ of both composites before post-cure increased compared to that of the pure MA composites, which suggested that a restriction of the polymer chain mobility was obtained through the addition of MMA-CNCs or CNCs into the MA matrix caused by the hydrogen bonds formed between the MMA-CNCs (or CNCs) and MA molecules (Kiziltas et al. 2015). However, the $T_{\mathrm{g}}$ decrease in both composites after post-cure was explained by the presence of MMA-CNCs (or CNCs), which formed hydrogen bonds in the interface to restrict the mobility of polymer chains, thus interrupting the dominant original interactions among the matrix polymer chains during post-cure (Liu et al. 2010; Xiang et al. 2017). The $T_{\mathrm{p}}$ of MMA-CNC/MA composites, both before and after post-cure, shifted to a higher temperature at $0.5 \%$ and $1 \%$ MMA-CNC contents compared to that of the pure MA composites. This shift in temperature indicated that the presence of MMA-CNCs facilitated the polymerization between the MMA-CNCs and MA molecules, which resulted in higher mechanical and thermal properties of the composites. However, due to the presence of CNCs, the dominant original interactions among the MA molecules were interrupted during 3D printing. Therefore, the unreacted molecules had further thermal polymerization at a lower temperature than that of pure MA (Table 1) during the DSC heating process and after post-cure. From the $\Delta H$ of both composites, it was confirmed that MMA-CNCs were more efficient than CNCs in promoting the polymerization of MA molecules with MMA$\mathrm{CNC}$ (or CNCs) because of the lower $\Delta H$ value of MMA-CNC/MA composites than that of CNC/MA composites both before and after post-cure.

Table 1. Glass Transition Temperature $\left(T_{g}\right)$, Thermal Polymerization

Temperature $\left(T_{\mathrm{p}}\right)$, and Enthalpy $(\Delta H)$ of MMA-CNC/MA and CNC/MA Composites Before and After Post-cure

\begin{tabular}{|c|c|c|c|c|c|c|}
\hline \multirow{2}{*}{ MMA-CNC (\%) } & \multicolumn{2}{|c|}{$\boldsymbol{T}_{\mathbf{g}}\left({ }^{\circ} \mathbf{C}\right)$} & \multicolumn{2}{c|}{$\boldsymbol{T}_{\mathbf{p}}\left({ }^{\circ} \mathbf{C}\right)$} & \multicolumn{2}{c|}{$\Delta \boldsymbol{H}\left(\mathbf{J} \cdot \mathbf{g}^{-1}\right)$} \\
\cline { 2 - 7 } & Before & After & Before & After & Before & After \\
\hline 0 & $45.3 \pm 0.1$ & $55.4 \pm 0.3$ & $165.0 \pm 0.5$ & $165.1 \pm 0.2$ & $-4.4 \pm 0.2$ & $-2.5 \pm 0.5$ \\
\hline 0.5 & $55.3 \pm 0.4$ & $49.8 \pm 0.1$ & $165.4 \pm 0.3$ & $165.7 \pm 0.4$ & $-7.4 \pm 0.4$ & $-5.5 \pm 0.2$ \\
\hline 1 & $54.9 \pm 0.3$ & $45.8 \pm 0.4$ & $168.9 \pm 0.1$ & $170.5 \pm 0.5$ & $-7.3 \pm 0.5$ & $-2.2 \pm 0.5$ \\
\hline 2 & $54.5 \pm 0.2$ & $39.3 \pm 0.1$ & $158.7 \pm 0.4$ & $160.9 \pm 0.2$ & $-11.8 \pm 0.5$ & $-4.2 \pm 0.1$ \\
\hline 4 & $45.1 \pm 0.3$ & $38.1 \pm 0.3$ & - & - & - & - \\
\hline CNC (\%) & $55.5 \pm 0.2$ & $47.9 \pm 0.3$ & $154.2 \pm 0.1$ & $164.7 \pm 0.2$ & $-13.9 \pm 0.2$ & $-1.1 \pm 0.3$ \\
\hline 0.5 & $56.5 \pm 0.5$ & $52.9 \pm 0.2$ & $154.3 \pm 0.2$ & $158.6 \pm 0.1$ & $-14.3 \pm 0.1$ & $-10.5 \pm 0.4$ \\
\hline 1 & $54.3 \pm 0.4$ & $47.1 \pm 0.1$ & $151.9 \pm 0.5$ & $156.4 \pm 0.4$ & $-14.7 \pm 0.3$ & $-4.5 \pm 0.2$ \\
\hline 2 & $53.3 \pm 0.2$ & $48.4 \pm 0.4$ & $139.5 \pm 0.2$ & $138.3 \pm 0.3$ & $-7.7 \pm 0.5$ & $-6.2 \pm 0.1$ \\
\hline 4 & \multicolumn{7}{|c|}{}
\end{tabular}




\section{CONCLUSIONS}

1. The cellulose nanocrystals (CNCs) modified with methylmethacyrylate (MMA) were incorporated in a methacrylate (MA) resin, and then the mixture was used to 3D print the composites. Compared to the unmodified CNCs, the modified CNCs exhibited better dispersion in the MA resin and less phase separation from the MA matrix because of the improvement in the interfacial compatibility.

2. This modification process improved the homogeneity of CNCs in the composites. Modification of CNCs with MMA improved the thermal stability of CNCs and the resulting $\mathrm{CNC} / \mathrm{MA}$ composites.

3. The reinforcement in the tensile property of the MA composite by the MMA-modified CNCs was substantially higher than that by the default CNC. In addition, MMA modification of CNCs resulted in improved thermal stability of the resulting MA composites, as evidenced by the TG and DSC results.

4. The study also showed that a post-cure process was essential to allow a complete polymerization reaction to occur in the composite. These findings demonstrated that modification of CNCs with MMA substantially improved the reinforcement efficiency of CNCs for the 3D-printed MA materials.

\section{ACKNOWLEDGEMENTS}

The authors are grateful the support of the National Key R \& D Program of China (Grant No. 2016YFD0600704) and the Priority Academic Program Development (PAPD) of Jiangsu Province, China. Financial support from the Soft Materials Research in Tennessee (SMART) and United States Department of Agriculture National Institute of Food and Agriculture (Hatch project No. 1012359) are gratefully acknowledged. The authors acknowledge support from the China Scholarship Council (No. 201506600011).

\section{REFERENCES CITED}

Anžlovar, A., Huskić, M., and Žagar, E. (2016). "Modification of nanocrystalline cellulose for application as a reinforcing nanofiller in PMMA composites," Cellulose 23(1), 505-518. DOI: 10.1007/s10570-015-0786-9

ASTM D638-14 (2014). "Standard test method for tensile properties of plastics," ASTM International, West Conshohocken, PA.

Becker, H., and Gärtner, C. (2008). "Polymer microfabrication technologies for microfluidic systems," Analytical and Bioanalytical Chemistry 390(1), 89-111. DOI: $10.1007 / \mathrm{s} 00216-007-1692-2$

Canche-Escamilla, G., Rodriguez-Laviada, J., Cauich-Cupul, J., Mendizabal, E., Puig, J., and Herrera-Franco, P. (2002). "Flexural, impact and compressive properties of a rigid-thermoplastic matrix/cellulose fiber reinforced composites," Composites Part A: Applied Science and Manufacturing 33(4), 539-549. DOI: 10.1016/S1359835X(01)00136-1

Chiappone, A., Bella, F., Nair, J. R., Meligrana, G., Bongiovanni, R., and Gerbaldi, C. 
(2014). "Structure-performance correlation of nanocellulose-based polymer electrolytes for efficient quasi-solid DSSCs," ChemElectroChem 1(8), 1350-1358. DOI: 10.1002/celc.201402051

Cooke, M. N., Fisher, J. P., Dean, D., Rimnac, C., and Mikos, A. G. (2003). "Use of stereolithography to manufacture critical-sized 3D biodegradable scaffolds for bone ingrowth," Journal of Biomedical Materials Research Part B: Applied Biomaterials 64B(2), 65-69. DOI: 10.1002/jbm.b.10485

Crivello, J. V., and Reichmanis, E. (2014). "Photopolymer materials and processes for advanced technologies," Chemistry of Materials 26(1), 533-548. DOI: $10.1021 / \mathrm{cm} 402262 \mathrm{~g}$

Dai Prè, M., Morrow, I., Martin, D. J., Mos, M., Del Negro, A., Padovani, S., and Martucci, A. (2013). "Preparation and characterization of down shifting ZnS: Mn/PMMA nanocomposites for improving photovoltaic silicon solar cell efficiency," Materials Chemistry and Physics 139(2-3), 531-536. DOI:

10.1016/j.matchemphys.2013.02.003

Dong, H., Strawhecker, K. E., Snyder, J. F., Orlicki, J. A., Reiner, R. S., and Rudie, A. W. (2012). "Cellulose nanocrystals as a reinforcing material for electrospun poly(methyl methacrylate) fibers: Formation, properties and nanomechanical characterization," Carbohydrate Polymers 87(4), 2488-2495. DOI: 10.1016/j.carbpol.2011.11.015

Esposito Corcione, C., Greco, A., and Maffezzoli, A. (2004). "Photopolymerization kinetics of an epoxy-based resin for stereolithography," Journal of Applied Polymer Science 92(6), 3484-3491. DOI: 10.1002/app.20347

Farahani, R. D., Dalir, H., Le Borgne, V., Gautier, L. A., El Khakani, M. A., Lévesque, M., and Therriault, D. (2012). "Direct-write fabrication of freestanding nanocomposite strain sensors," Nanotechnology 23(8), 085502-085600. DOI: 10.1088/0957-4484/23/8/085502

Feng, X., Yang, Z., Chmely, S., Wang, Q., Wang, S., and Xie, Y. (2017). "Lignin-coated cellulose nanocrystal filled methacrylate composites prepared via $3 \mathrm{D}$ stereolithography printing: Mechanical reinforcement and thermal stabilization," Carbohydrate Polymers 169, 272-281. DOI: 10.1016/j.carbpol.2017.04.001

Feng, X., Yang, Z., Rostom, S. S. H., Dadmun, M., Wang, S., Wang, Q., and Xie, Y. (2018). "Reinforcing 3D printed acrylonitrile butadiene styrene by impregnation of methacrylate resin and cellulose nanocrystal mixture: Structural effects and homogeneous properties," Materials \& Design 138, 62-70. DOI:

10.1016/j.matdes.2017.10.050

Kedzior, S. A., Graham, L., Moorlag, C., Dooley, B. M., and Cranston, E. D. (2016). "Poly(methyl methacrylate)-grafted cellulose nanocrystals: One-step synthesis, nanocomposite preparation, and characterization," The Canadian Journal of Chemical Engineering 94(5), 811-822. DOI: 10.1002/cjce.22456

Kim, K., Zhu, W., Qu, X., Aaronson, C., McCall, W. R., Chen, S., and Sirbuly, D. J. (2014). "3D optical printing of piezoelectric nanoparticle-polymer composite materials," ACS Nano 8(10), 9799-9806. DOI: 10.1021/nn503268f

Kiziltas, E. E., Kiziltas, A., Bollin, S. C., and Gardner, D. J. (2015). "Preparation and characterization of transparent PMMA-cellulose-based nanocomposites," Carbohydrate Polymers 127, 381-389. DOI: 10.1016/j.carbpol.2015.03.029

Kramer, F., Klemm, D., Schumann, D., Heßler, N., Wesarg, F., Fried, W., and Stadermann, D. (2006). "Nanocellulose polymer composites as innovative pool for (bio) material development," Macromolecular Symposia 244(1), 136-148. DOI: 
10.1002/masy.200651213

Kuo, S.-W. (2008). "Hydrogen-bonding in polymer blends," Journal of Polymer Research 15(6), 459-486. DOI: 10.1007/s10965-008-9192-4

Lee, K. -S., Kim, R. H., Yang, D. -Y., and Park, S. H. (2008). "Advances in 3D nano/microfabrication using two-photon initiated polymerization," Progress in Polymer Science 33(6), 631-681. DOI: 10.1016/j.progpolymsci.2008.01.001

Lin, D., Jin, S., Zhang, F., Wang, C., Wang, Y., Zhou, C., and Cheng, G. J. (2015). "3D stereolithography printing of graphene oxide reinforced complex architectures," Nanotechnology 26(43), Article ID 434003. DOI: 10.1088/0957-4484/26/43/434003

Lin, N. J., Bailey, L. O., Becker, M. L., Washburn, N. R., and Henderson, L. A. (2007). "Macrophage response to methacrylate conversion using a gradient approach," Acta Biomaterialia 3(2), 163-173. DOI: 10.1016/j.actbio.2006.10.001

Littunen, K., Hippi, U., Johansson, L. -S., Österberg, M., Tammelin, T., Laine, J., and Seppälä, J. (2011). "Free radical graft copolymerization of nanofibrillated cellulose with acrylic monomers," Carbohydrate Polymers 84(3), 1039-1047. DOI:

10.1016/j.carbpol.2010.12.064

Littunen, K., Hippi, U., Saarinen, T., and Seppälä, J. (2013). "Network formation of nanofibrillated cellulose in solution blended poly(methyl methacrylate) composites," Carbohydrate Polymers 91(1), 183-190. DOI: 10.1016/j.carbpol.2012.08.032

Liu, H., Liu, D., Yao, F., and Wu, Q. (2010). "Fabrication and properties of transparent polymethylmethacrylate/cellulose nanocrystals composites," Bioresource Technology 101(14), 5685-5692. DOI: 10.1016/j.biortech.2010.02.045

Mao, Y., Miyazaki, T., Sakai, K., Gong, J., Zhu, M., and Ito, H. (2018). "A 3D printable thermal energy storage crystalline gel using mask-projection stereolithography," Polymers 10(10), 1117-1130. DOI: 10.3390/polym10101117

Melchels, F. P., Feijen, J., and Grijpma, D. W. (2010). "A review on stereolithography and its applications in biomedical engineering," Biomaterials 31(24), 6121-6130. DOI: 10.1016/j.biomaterials.2010.04.050

Moon, S. K., Tan, Y. E., Hwang, J., and Yoon, Y. -J. (2014). "Application of 3D printing technology for designing light-weight unmanned aerial vehicle wing structures," International Journal of Precision Engineering and Manufacturing-Green Technology 1(3), 223-228. DOI: 10.1007/s40684-014-0028-x

Nguyen, L., Straub, M., and Gu, M. (2005). "Acrylate-based photopolymer for twophoton microfabrication and photonic applications," Advanced Functional Materials 15(2), 209-216. DOI: 10.1002/adfm.200400212

Qin, D., Xia, Y., and Whitesides, G. M. (2010). "Soft lithography for micro- and nanoscale patterning," Nature Protocols 5(3), 491-502. DOI: 10.1038/nprot.2009.234

Routray, C., and Tosh, B. (2012). "Controlled grafting of MMA onto cellulose and cellulose acetate," Cellulose 19(6), 2115-2139. DOI: 10.1007/s10570-012-9762-9

Sain, S., Ray, D., and Mukhopadhyay, A. (2015). "Improved mechanical and moisture resistance property of in situ polymerized transparent PMMA/cellulose composites," Polymer Composites 36(9), 1748-1758. DOI: 10.1002/pc.23102

Sain, S., Ray, D., Mukhopadhyay, A., Sengupta, S., Kar, T., Ennis, C. J., and Rahman, P. K. (2012). "Synthesis and characterization of PMMA-cellulose nanocomposites by in situ polymerization technique," Journal of Applied Polymer Science 126(S1), E127E134. DOI: 10.1002/app.36723

Schaedler, T. A., and Carter, W. B. (2016). "Architected cellular materials," Ann. Review of Materials Research 46, 187-210. DOI: 10.1146/annurev-matsci-070115-031624 
Shanmuganathan, K., Capadona, J. R., Rowan, S. J., and Weder, C. (2009). "Stimuliresponsive mechanically adaptive polymer nanocomposites," ACS Applied Materials \& Interfaces 2(1), 165-174. DOI: 10.1021/am9006337

Singha, A. S., and Thakur, V. K. (2009). "Chemical resistance, mechanical and physical properties of biofibers-based polymer composites," Polymer-Plastics Technology and Engineering 48(7), 736-744. DOI: 10.1080/03602550902824622

Suntornnond, R., An, J., and Chua, C. K. (2017). "Bioprinting of thermoresponsive hydrogels for next generation tissue engineering: A review," Macromolecular Materials and Engineering 302(1), Article ID 1600266. DOI:

10.1002/mame.201600266

Tran, T. N., Paul, U., Heredia-Guerrero, J. A., Liakos, I., Marras, S., Scarpellini, A., Ayadi, F., Athanassiou, A., and Bayer, I. S. (2016). "Transparent and flexible amorphous cellulose-acrylic hybrids," Chemical Engineering Journal 287, 196-204. DOI: $10.1016 /$ j.cej.2015.10.114

Tsai, C. -W., Wang, J. -C., Li, F. -N., Chang, Y. -C., and Wu, K. -H. (2016). "Synthesis of adamantane-containing methacrylate polymers: Characterization of thermal, mechanical, dielectric and optical properties," Materials Express 6(3), 220-228. DOI: 10.1166/mex.2016.1298

Vatani, M., Lu, Y., Engeberg, E. D., and Choi, J. -W. (2015). "Combined 3D printing technologies and material for fabrication of tactile sensors," International Journal of Precision Engineering and Manufacturing 16(7), 1375-1383. DOI: 10.1007/s12541015-0181-3

Wan, Z., Xiong, Z., Ren, H., Huang, Y., Liu, H., Xiong, H., Wu, Y., and Han, J. (2011). "Graft copolymerization of methyl methacrylate onto bamboo cellulose under microwave irradiation," Carbohydrate Polymers 83(1), 264-269. DOI:

10.1016/j.carbpol.2010.07.048

Weng, Z., Zhou, Y., Lin, W., Senthil, T., and Wu, L. (2016). "Structure-property relationship of nano enhanced stereolithography resin for desktop SLA 3D printer," Composites Part A: Applied Science and Manufacturing 88, 234-242. DOI: 10.1016/j.compositesa.2016.05.035

Xiang, H., Wang, X., Lin, G., Xi, L., Yang, Y., Lei, D., Dong, H., Su, J., Cui, Y., and Liu, X. (2017). "Preparation, characterization and application of UV-curable flexible hyperbranched polyurethane acrylate," Polymers 9(11), 552-563. DOI: 10.3390/polym9110552

$\mathrm{Xu}, \mathrm{K}$., and Chen, Y. (2015). "Mask image planning for deformation control in projection-based stereolithography process," Journal of Manufacturing Science and Engineering 137(3), Article ID 031014. DOI: 10.1115/1.4029802

Yang, Z., Wu, G., Wang, S., Xu, M., and Feng, X. (2018). "Dynamic postpolymerization of 3D-printed photopolymer nanocomposites: Effect of cellulose nanocrystal and postcure temperature," Journal of Polymer Science Part B: Polymer Physics 56(12), 935-946. DOI: 10.1002/polb.24610

Zhang, X., Jiang, X., and Sun, C. (1999). "Micro-stereolithography of polymeric and ceramic microstructures," Sensors and Actuators A: Physical 77(2), 149-156. DOI: 10.1016/S0924-4247(99)00189-2

Article submitted: January 18, 2019; Peer review completed: March 18, 2019; Revisions accepted: March 19, 2019; Published: March 20, 2019.

DOI: 10.15376/biores.14.2.3701-3716 